\title{
Decomposition of dextran sulfate sodium under alkaline, acidic, high temperature and high pressure conditions
}

\author{
YOSHIO ARAKI, KEN-ICHI MUKAISYO, HIROYUKI SUGIHARA and TAKANORI HATTORI
}

\author{
Department of Pathology, Shiga University of Medical Science, Seta Tsukinowa, Otsu 520-2192, Shiga, Japan
}

Received December 20, 2007; Accepted March 18, 2008

\begin{abstract}
The pathogenic mechanisms responsible for inflammatory bowel disease (IBD) are poorly understood. In an IBD animal model, the oral administration of polysaccharides such as dextran sulfate sodium (DSS) induced colitis, which exhibited several clinical and histological features similar to IBD. However, pathogenic factors in the development of colitis remain unclear, as do the chemical properties of DSS. Therefore, we investigated the chemical properties of DSS in this study. DSS reacted under $1 \mathrm{~N}$ acidic or alkaline conditions, and the reactants were analyzed using size exclusion and ion chromatography. The reactants were also analyzed in terms of metachromasia as a peculiarity of sulfated polysaccharides. DSS was depolymerized to a $M r 2500$ moiety under acidic, but not under alkaline conditions. Sulfate was depleted from DSS under alkaline conditions $(30 \%)$, but less so under acidic conditions $(10 \%)$. In addition, autoclave treatment induced depolymerization to a $M r 1800$ moiety and depleted the sulfate. This depolymerized DSS under acidic conditions has as many sulfates as a native DSS molecule with a $\mathrm{Mr}$ of 5000 , exhibiting metachromasia as a peculiarity of sulfated polysaccharides. Therefore, these $M r 2500$ or 1800 DSS molecules may be useful in investigating the mechanisms of DSS-induced colitis.
\end{abstract}

\section{Introduction}

Human inflammatory bowel disease (IBD), including Crohn's disease (CD) as well as ulcerative colitis (UC), is a chronic, relapsing and remitting condition that exhibits several features of immunological inflammation. IBD is characterized by an infiltration of large numbers of neutrophils, monocytes and lymphocytes into the large and/or small intestine causing diarrhea, bloody stools, abdominal pain, body weight loss, anemia and leukocytosis. Despite many investigations, the etiology and pathogenic mechanisms responsible for IBD

Correspondence to: Dr Yoshio Araki, 6-13-20 Aoyama, Otsu, Shiga 520-2101, Japan

E-mail: yir0828@belle.shiga-med.ac.jp

Key words: dextran sulfate sodium, decomposition, sulfate, depolymerization, acidic conditions, high temperature, high pressure remain poorly understood. However, experimental animal models of IBD are being developed to investigate its pathogenesis, and to improve treatment options. Most commonly, colitis is induced by administering polysaccharides such as dextran sulfate sodium (DSS) or carrageenan. DSS is a heparin-like polysaccharide containing $\sim 17 \%$ sulfur with up to three sulfates per glucose molecule (Fig. 1) (1). With respect to the pathogenic factors in this colitis model, previous reports have postulated the importance of various factors such as local immunological disturbances (2), reactive oxygen species $(\operatorname{ROS})(3,4)$ and short chain fatty acids $(5)$. However, the precise mechanisms responsible for DSS-induced colitis remain unclear, as do the chemical properties of DSS. Therefore, we investigated some of the chemical properties of DSS in this study.

\section{Materials and methods}

Chemicals. DSS (Mr 5000), 2-aminopyridine and tetra-nbutylammonium bromide were obtained from Wako Pure Chemical Industries, Ltd. (Osaka, Japan). DSS ( $M r 8000$ or 10000) and D-glucose 3-sulfate were obtained from Sigma (Aldrich) Chemical Co. (St. Louis, MO).

Labeling of DSS. The molecular mass of DSS and D-glucose 3 -sulfate was labeled using 2-aminopyridine according to our previously reported method $(6,7)$.

Decomposition of dextran sulfate sodium under alkaline and acidic conditions, or by autoclave treatment. DSS (Mr 5000) and pyridylamino-DSS (PA-DSS) were dissolved in $1 \mathrm{~N}$ acidic or alkaline solutions to $3 \%$ and was incubated at $37^{\circ} \mathrm{C}$. After the samples were neutralized, a $10 \mu 1$ aliquot of the reactants was analyzed. In addition, 3\% DSS was also autoclaved at $115^{\circ} \mathrm{C}$ for $15 \mathrm{~min}(1.7 \mathrm{~atm})$.

HPLC conditions. In the present size exclusion chromatography (SEC) system, we used an HPLC LC6A apparatus (Shimadzu, Kyoto, Japan), a Cosmosil 5Diol-120 packed column (7.5x300 mm, Nacalai Tesque Inc., Kyoto, Japan) maintained at $60^{\circ} \mathrm{C}$ and a fluorescence detector RF-535 (Shimadzu) with excitation and emission wavelengths of 320 and $400 \mathrm{~nm}$, respectively. The mobile phase consisted of $0.2 \mathrm{M}$ phosphate buffer $(\mathrm{pH} 3.0)$ at $1.0 \mathrm{ml} / \mathrm{min}$.

In the ion chromatography (IC) system, we used an IonPac AS12A column (4x200 mm, Dionex Co., Sunnyvale, CA) with an ion conductivity detector. The mobile phase consisted of a 


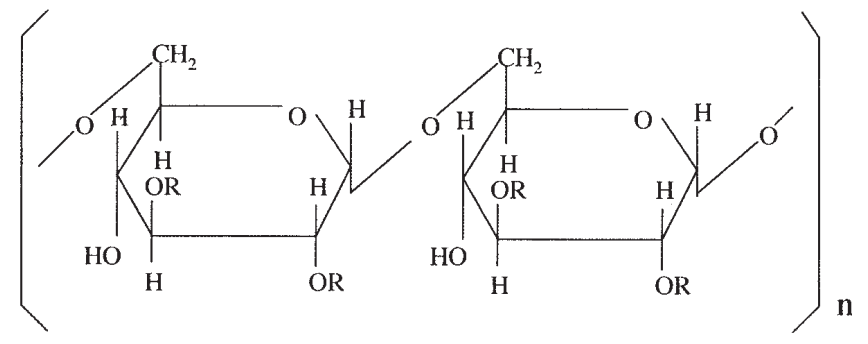

R: $\mathrm{SO}_{3} \mathrm{Na}$ or $\mathrm{H}$

Figure 1. Structure of dextran sulfate sodium.

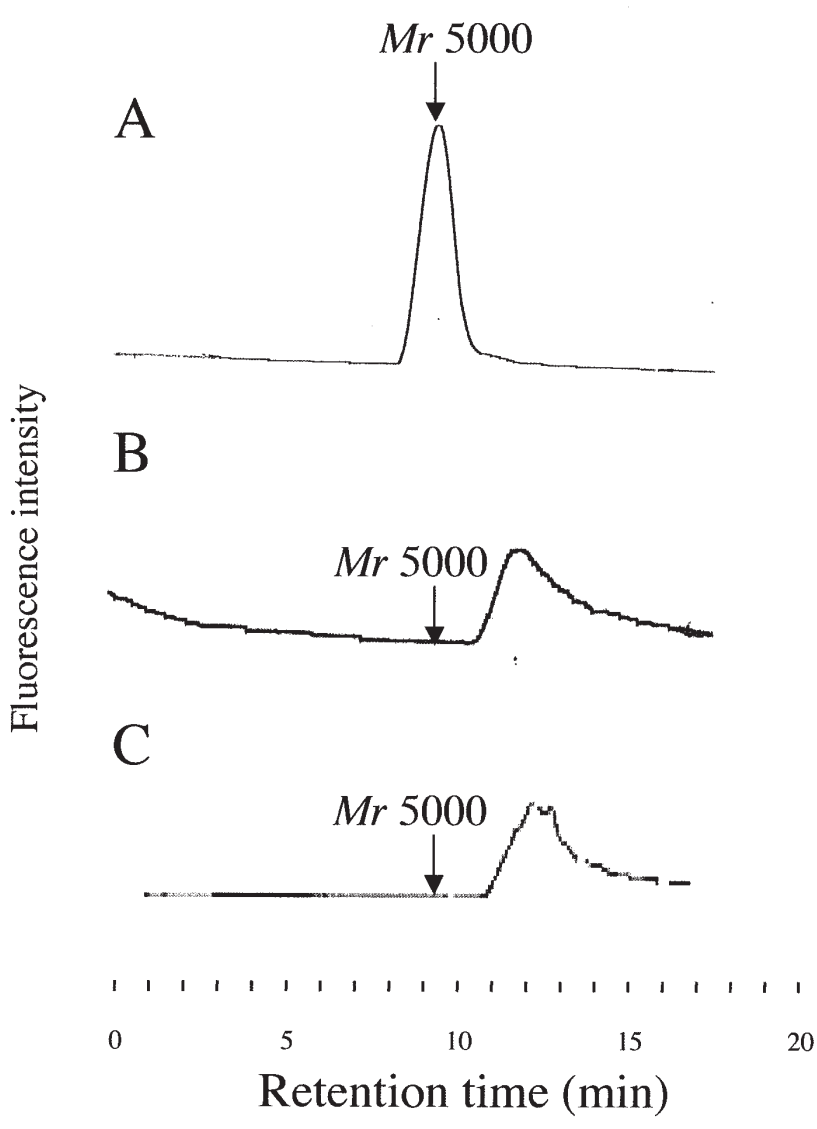

Figure 2. Size exclusion chromatogram of pyridylamino-dextran sulfate (PA-DSS) and the depolymerization process. (A) The native PA-DSS shows one peak at $M r$ 5000. (B) In a $1 \mathrm{~N} \mathrm{HCl}$ solution, the PA-DSS was depolymerized to $\sim M r 2500$ on day 3. (C) The PA-DSS was depolymerized to $M r 1800$ following autoclave treatment. The arrows represent $M r 5000$. The HPLC conditions are described in the Materials and methods.

$2 \mathrm{mM}$ sodium carbonate/1 $\mathrm{mM}$ sodium hydrogen carbonate solution at $1.5 \mathrm{ml} / \mathrm{min}$. The suppressor was AMMS-III (Dionex).

Metachromasia. In general, a mixture of DSS solution and toluidine blue solution exhibits a color change from blue to reddish purple, a phenomenon called metachromasia (8). We investigated whether these reactants exhibited metachromasia. A $100 \mu \mathrm{l}$ aliquot from the reactants was added to $2 \mathrm{ml}$

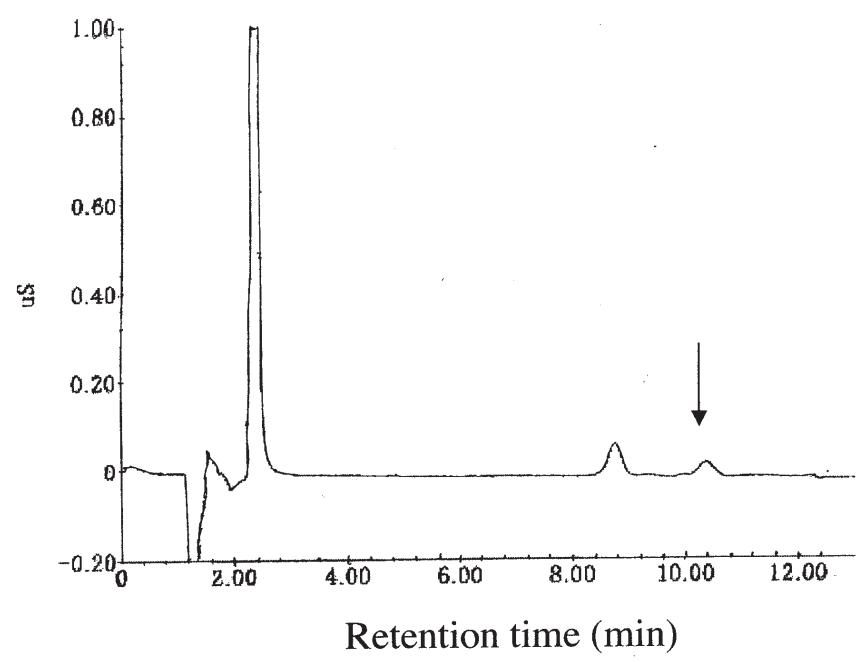

Figure 3. Ion-exchange chromatogram of the process of sulfate release from DSS. After 3 days under acidic conditions, the concentration of sulfate increased to $0.17 \%$. The arrow represents the sulfate group. The HPLC conditions are described in the Materials and methods.

of $0.01 \%$ toluidine blue solution. The mixture was then analyzed using an Ultrospec 3000 (Amersham, USA) for spectrophotometric absorbance measurements.

\section{Results}

Decomposition under alkaline or acidic conditions, or autoclave treatment. Fig. 2 shows the SEC of native PA-DSS and the depolymerization process. Native PA-DSS showed one peak at $\mathrm{Mr} 5000$ (Fig. 2A). In a $1 \mathrm{~N} \mathrm{HCl}$ solution, PADSS was depolymerized by day 3 (Fig. 2B). At this time, the molecular mass was speculated to be $\sim 2500$ according to the relationship between the elution time and the molecular mass of each PA-DSS and PA-D-glucose 3-sulfate moiety.

Under alkaline conditions, however, even a $7 \mathrm{~N} \mathrm{NaOH}$ solution did not depolymerize DSS after a 3-day incubation (data not shown). On the other hand, PA-DSS was depolymerized to $\mathrm{Mr} 1800$ following autoclave treatment (Fig. 2C).

Fig. 3 shows the IC of the processes of sulfate release from DSS. The concentration of sulfate in the $3 \%$ native DSS solution was $\sim 0.014 \%$. After 3 days under acidic conditions, the concentration of sulfate increased to $0.17 \%$, meaning that $10 \%$ of the sulfate groups were depleted from one DSS molecule (allow). Alkaline conditions depleted more sulfate from DSS than the acidic conditions ( $\sim 3$-fold more) (data not shown). Sulfate depletion was also more recognized following autoclave treatment ( $\sim$-fold more) (data not shown).

Metachromasia. In toluidine blue solution, a large peak of UV absorbance was observed at $620 \mathrm{~nm}$. This large peak shifted to $560 \mathrm{~nm}$ when the metachromasia occurred (8). Metachromasia was observed in native $\mathrm{Mr} 5000$ and 2500 DSS, depolymerized under acidic conditions. However, metachromasia was no longer observed in the reactants under alkaline conditions or following autoclave treatment. These results suggest that metachromasia in the DSS solution requires a sufficiently high sulfate content. 


\section{Discussion}

In our previous study, we reported that the early stages of DSS-induced colitis involved cytotoxic effects to the epithelial cells rather than immunological or fibrolytic mechanisms. DSS entered the cells and bound to the nucleus in Caco- 2 cells. Thereafter, DSS induced cell cycle arrest and inhibited ROS generation. Intestinal epithelial cells have a rapid cell cycle. Therefore, cell cycle arrest may lead to breaks in the barrier and absorptive functions of these cells (9).

The chemical properties of DSS remain unclear. We investigated the chemical properties of DSS, including its reactants, under acidic and alkaline conditions using SEC and IC. It has become clear that DSS was depolymerized to a $M r 2500$ moiety under acidic, but not under alkaline conditions. In addition, autoclave treatment depolymerized DSS to $M r 1800$. However, the sulfate was dramatically depleted from DSS under alkaline conditions (30\%), but less so under acidic conditions or after autoclave treatment $(<10 \%)$. Sulfate depletion was more recognized following autoclave treatment $(70 \%)$, indicating that autoclave treatment for sterilization induced the decomposition of DSS.

These novel $M r 2500$ moieties may be useful in investigating the mechanisms of DSS-induced colitis in animals, because they have as many sulfate groups in a molecule as native $M r 5000$ DSS. Furthermore, it has become clear that metachromasia is dependent on the sulfate content of DSS.

In conclusion, we found that acidic conditions produced Mr 2500 moieties from native $M r 5000$ DSS, without losing any sulfate. This novel $M r 2500$ DSS moieties may be useful in investigating the mechanisms of DSS-induced colitis.

\section{Acknowledgements}

We thank Mr. Noboru Urushiyama of Central Research Laboratory, Shiga University of Medical Science for his assistance with the HPLC analyses.

\section{References}

1. Ricketts CR: Dextran sulphate - a synthetic analogue of heparin. Biochem J 51: 129-133, 1952.

2. Kobayashi K, Asakura H, Hamada Y, Hibi T, Watanabe M, Yoshida T, Watanabe N, Miura S, Aiso S and Tsuchiya M: T lymphocyte subpopulations and immunoglobulin-containing cells in the colonic mucosa of ulcerative colitis; a morphometric and immunohistochemical study. J Clin Lab Immunol 25: 63-68, 1988.

3. Araki Y, Andoh A and Fujiyama Y: The free radical scavenger edaravone suppresses experimental dextran sulfate sodiuminduced colitis in rats. Int J Mol Med 12: 125-129, 2003.

4. Araki Y, Sugihara H and Hattori T: The free radical scavengers edaravone and tempol suppress experimental dextran sulfate sodium-induced colitis in mice. Int J Mol Med 17: 331-334, 2006.

5. Araki Y, Andoh A, Takizawa J, Takizawa W and Fujiyama Y: Clostridium butyricum, a probiotic derivative, suppresses dextran sulfate sodium-induced experimental colitis in rats. Int J Mol Med 13: 577-580, 2004.

6. Araki Y, Andoh A, Fujiyama Y, Hata K, Makino J, Okuno T, Nakanura F and Bamba T: Application of 2-aminopyridine fluorescence labeling in the analysis of in vivo and in vitro metabolism of dextran sulfate sodium by size-exclusion highperformance liquid chromatography. J Chromatogr B Biomed Sci Appl 753: 209-215, 2001

7. Araki Y, Andoh A, Fujiyama Y, Hata K, Makino J, Shimada M, Bamba H, Okuno T, Urushiyama $\mathrm{N}$ and Bamba T: Analysis of $\alpha$-amylase-derived pyridylamino-dextran sulfate oligomers by the combination of size-exclusion and reversed-phase highperformance liquid chromatography. J Chromatogr B Biomed Sci Appl 766: 351-356, 2002.

8. Araki Y, Katoh T, Urabe M, Kishi Y, Ishizuka I and Fujiyama Y: The analysis of pyridylamino-dextran sulfate oligomers by highperformance liquid chromatography and a novel detection system for sulfated polysaccharides. Oncol Rep 12: 363-367, 2004.

9. Araki $\mathrm{Y}$, Sugihara $\mathrm{H}$ and Hattori $\mathrm{T}$ : In vitro effects of dextran sulfate sodium on a Caco- 2 cell line and plausible mechanisms for dextran sulfate sodium-induced colitis. Oncol Rep 16: 1357-1362, 2006. 